\title{
Un épanchement pleural atypique
}

\section{An atypical pleural effusion}

\section{A. Prost $\cdot$ Y. Zlakomanov $\cdot$ P. Vallaghe}

Reçu le 8 janvier 2012 ; accepté le 4 février 2012

(C) SFMU et Springer-Verlag France 2012
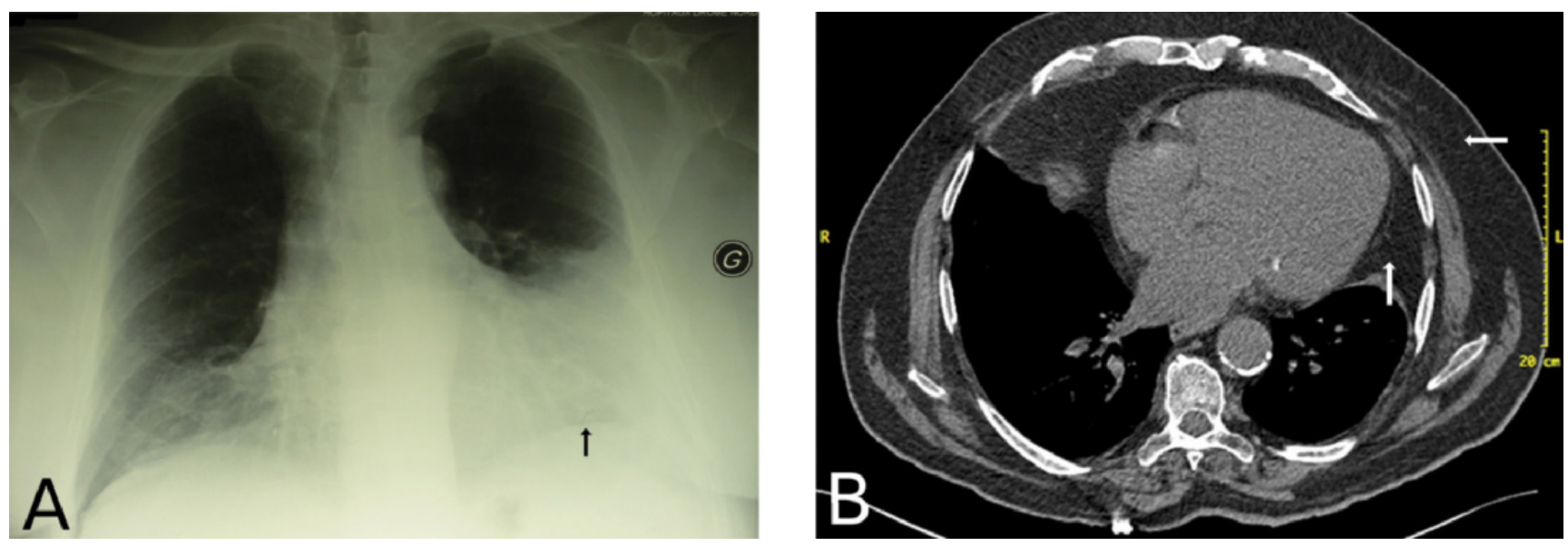

Fig. 1 Radiographie pulmonaire de face (A), la flèche souligne la coupole diaphragmatique gauche, remettant en cause le diagnostic d'épanchement liquidien. Coupe scannographique thoracique (B) objectivant la LM, de densité graisseuse, comme le tissu sous cutané extra thoracique adjacent (flèches blanches)

Un patient de 80 ans, obèse, ayant une bronchopneumopathie chronique post-tabagique consulte pour une douleur basi-thoracique gauche, persistant 10 jours après une chute. La radiographie pulmonaire montre une opacité d'allure pleurale de l'hémichamp pulmonaire gauche (Fig. 1A). Toutefois, le diagnostic d'épanchement liquidien est infirmé car l'opacité efface la pointe du cœur mais pas la coupole diaphragmatique (flèche noire). Un scanner thoracique (Fig. 1B) montre une volumineuse lipomatose médiastinale (LM) à extension antéro-basale bilatéral. La LM, pathologie bénigne, est caractérisée par une abondante quantité de graisse dans le médiastin, souvent secondaire à une corticothérapie, une obésité, ou un alcoolisme, de découverte souvent fortuite et se compliquant rarement. Il s'agit souvent d'un simple élargissement de la silhouette médiastinale, source d'erreurs diagnostiques [1].

\section{Référence}

1. Lê LH, Nguyên KQ, Phan HT, Hoeffel C (1998) Mediastinal lipomatosis. South Med J 91:1169-72

\footnotetext{
A. Prost $(\bowtie) \cdot$ Y. Zlakomanov

Service des urgences, Centre hospitalier de Romans-sur-Isère, BP102, F-26102 Romans Cedex, France

e-mail : anthonyprost@hotmail.com

P. Vallaghe

Pôle cardio-pneumologie, Centre hospitalier de Romans-sur-Isère, BP102, F-26102 Romans Cedex, France
} 